EPJ Web of Conferences 106, 01001 (2016)

DOI: $10.1051 /$ epjconf/201610601001

(C) Owned by the authors, published by EDP Sciences, 2016

\title{
Characterization of Novel Calorimeters in the Annular Core Research Reactor ${ }^{a}$
}

\author{
Brian D. Hehr ${ }^{1, b}$, Edward J. Parma ${ }^{1}$, Curtis D. Peters ${ }^{2}$, Gerald E. Naranjo ${ }^{2}$, and S. Michael Luker ${ }^{1}$ \\ ${ }^{1}$ Sandia National Laboratories, Applied Nuclear Technologies Department, P.O. Box 5800, \\ Albuquerque, New Mexico 87185, USA \\ 2 Sandia National Laboratories, Advanced Nuclear Concepts Department, P.O. Box 5800, Albuquerque, \\ New Mexico 87185, USA
}

\begin{abstract}
A series of pulsed irradiation experiments have been performed in the central cavity of Sandia National Laboratories' Annular Core Research Reactor (ACRR) to characterize the responses of a set of elemental calorimeter materials including $\mathrm{Si}, \mathrm{Zr}, \mathrm{Sn}$, $\mathrm{Ta}, \mathrm{W}$, and $\mathrm{Bi}$. Of particular interest was the perturbing effect of the calorimeter itself on the ambient radiation field - a potential concern in dosimetry applications. By placing the calorimeter package into a neutron-thermalizing lead/polyethylene (LP) bucket and irradiating both with and without a cadmium wrapper, it was demonstrated that prompt capture gammas generated inside the calorimeters can be a significant contributor to the measured dose in the active disc region. An MCNP model of the experimental setup was shown to replicate measured dose responses to within $10 \%$. The internal $(\mathrm{n}, \gamma)$ contribution was found to constitute as much as $50 \%$ of the response inside the LP bucket and up to $20 \%$ inside the nominal (unmodified) cavity environment, with $\mathrm{Ta}$ and $\mathrm{W}$ exhibiting the largest enhancement due to their sizable $(\mathrm{n}, \gamma)$ cross sections. Capture reactions in non-disc components of the calorimeter were estimated to be responsible for up to a few percent of the measured response.
\end{abstract}

\section{Introduction}

Calorimetry has proven to be a useful dosimetry technique in pulsed irradiation experiments at watermoderated reactors of a relatively long (millisecond-order) pulse width, such as the Annular Core Research Reactor (ACRR) [1], and also fast-burst assemblies of a much shorter (microsecond-order) pulse width such as the White Sands Missile Range fast burst reactor known as MoLLY-G [2]. Since calorimeters gauge a rather fundamental property - i.e. the heating induced by radiation interactionscalibration exercises are largely unnecessary and virtually any material is intrinsically viable as a calorimetric sensor, which typically takes the form of a small disc. Consequently, the selection of the

\footnotetext{
${ }^{a}$ This work was supported by the United States Department of Energy under Contract DE-AC04-94AL85000. Sandia is a multiprogram laboratory operated by Sandia Corporation, a Lockheed Martin Company, for the United States Department of Energy.

${ }^{\mathrm{b}}$ Corresponding author: bdhehr@sandia.gov
}

This is an Open Access article distributed under the terms of the Creative Commons Attribution License 2.0, which permits unrestricted use, distribution, and reproduction in any medium, provided the original work is properly cited. 
sensor material may be tailored to the desired sensitivity (e.g. gammas versus neutrons, slow neutrons versus fast neutrons, etc.).

Calorimeters are normally incorporated into an experimental package for the purpose of quantifying the radiation environment as would impinge upon a test article at the same location (or at a location equivalent by symmetry). In practice, the active disc, or even the sub-structure of the calorimeter, can perturb the measured signal to a considerable extent due to the internal production of secondary radiation. It is therefore important to understand the magnitude of these perturbations under realistic irradiation conditions.

A series of experiments were performed in the central cavity of ACRR to characterize the responses of a set of elemental $\mathrm{Si}, \mathrm{Zr}, \mathrm{Sn}, \mathrm{Ta}, \mathrm{W}$, and Bi calorimeter discs. The goals of this study were:

1) To investigate differences in response behavior among the various materials, including that portion attributable to neutron capture reactions within the calorimeters.

2) To assess the fidelity of an MCNP model in replicating the observed responses.

3) To survey the apportionment and origin of significant radiative dose contributions.

The experimental setup, described in the following section, was designed with the aim of maximizing the degree of thermalization of the neutrons reaching the calorimeter array. This was done to provide a strong signal from secondary radiation in order to validate that component of the response.

\section{Experimental Setup}

ACRR is a water-moderated, pool type reactor rated at a peak steady-state power of $4 \mathrm{MW}_{\text {th. }}$ By rapidly ejecting a set of transient rods, ACRR can be pulsed to a maximum energy of $300 \mathrm{MJ}$ with an associated pulse FWHM of approximately 7 milliseconds - energetically equivalent to a mean power of tens of thousands of megawatts during the pulse. A distinguishing feature of ACRR is the presence of a $9^{\prime \prime}$ diameter dry cavity extending the full length of the pool and into the core, allowing for the convenient insertion of experimental packages for irradiation. While the neutron spectrum in the vicinity of mid-core is nominally epithermal, several spectrum-modifying buckets are available for the purpose of emphasizing or de-emphasizing particular spectral components.

In order to maximize the calorimeter signal component originating from $(n, \gamma)$ reactions, a leadpolyethylene bucket was inserted into the central cavity. This bucket, composed of $0.4^{\prime \prime}(1.02 \mathrm{~cm})$ of lead and $0.8^{\prime \prime}(2.03 \mathrm{~cm})$ of HDPE encased in an aluminum liner, generates a highly thermalized neutron environment as illustrated in Fig. 1.

A staggered array of 10 calorimeters (including redundancies of certain types), shown in Fig. 2, was attached to an aluminum spindle designed to fit securely within the lead/poly bucket. Next, a sequence of pulsed irradiations was conducted with and without a cadmium wrapper surrounding the calorimeter array. The cadmium wrapper - which measured $43 / 4^{\prime \prime}$ in diameter, $4^{\prime \prime}$ in height, and 30 mils $(0.76 \mathrm{~mm})$ in thickness - was supported by an aluminum stand connected to the spindle (see righthand panel of Fig. 2). By blocking out most calorimeter $(n, \gamma)$ reactions below the cadmium cutoff energy without substantially altering other response components, the wrapper provided a means of separating out the calorimeter prompt $(\mathrm{n}, \gamma)$ signal from the ambient radiation-driven signal.

The calorimeters utilized in this experiment produced measurable data by means of the Seeback effect, by which a temperature differential between the junctions of two dissimilar conductors generates an electromotive force. Specifically, a type E thermocouple comprised of constantan $(55 \% \mathrm{Cu}-45 \%$ $\mathrm{Ni}$ ) braided with chromel $(10 \% \mathrm{Cr}-90 \% \mathrm{Ni})$ was deployed between the data acquisition station and the active region (disc) of the calorimeter. 


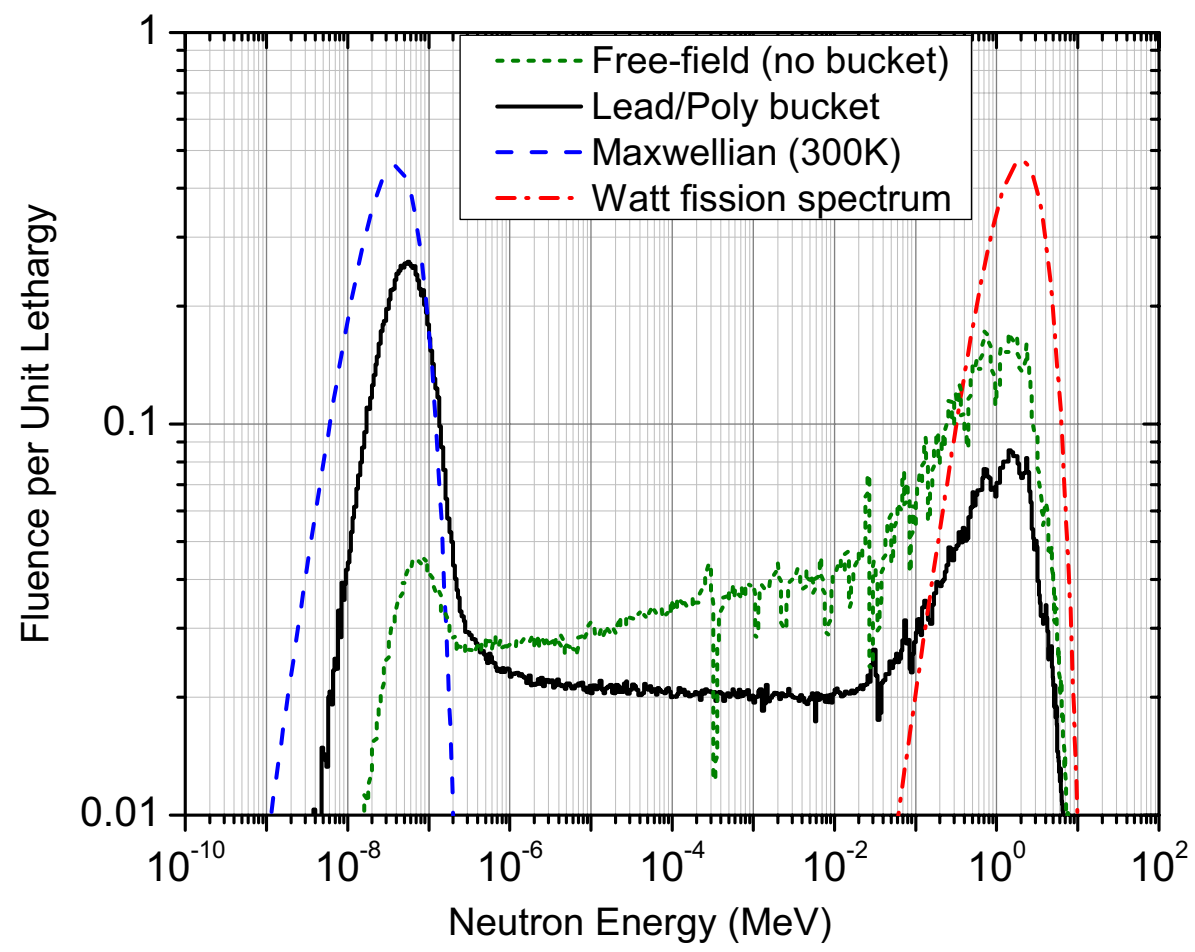

Figure 1. Neutron spectra in the ACRR central cavity.

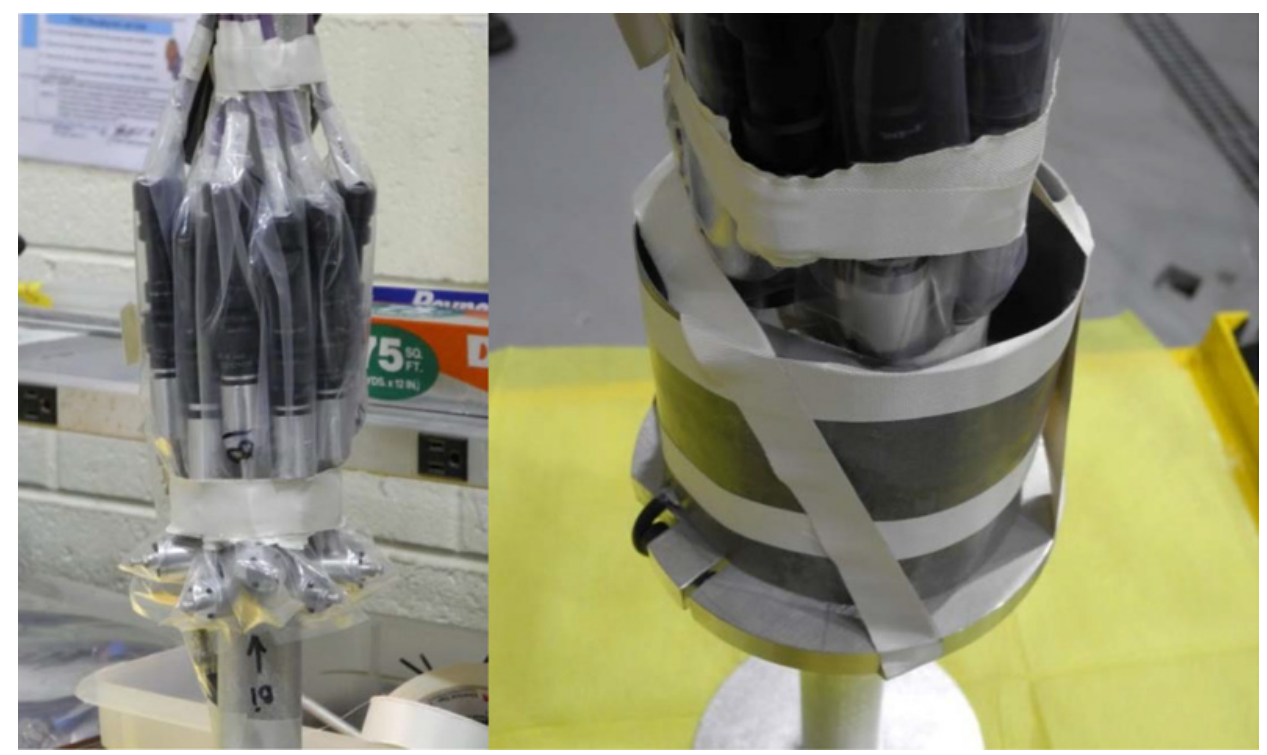

Figure 2. Bare calorimeter assembly on aluminum spindle (left panel) and the same assembly with a 30-mil $(0.76 \mathrm{~mm})$ cadmium wrapper (right panel). 


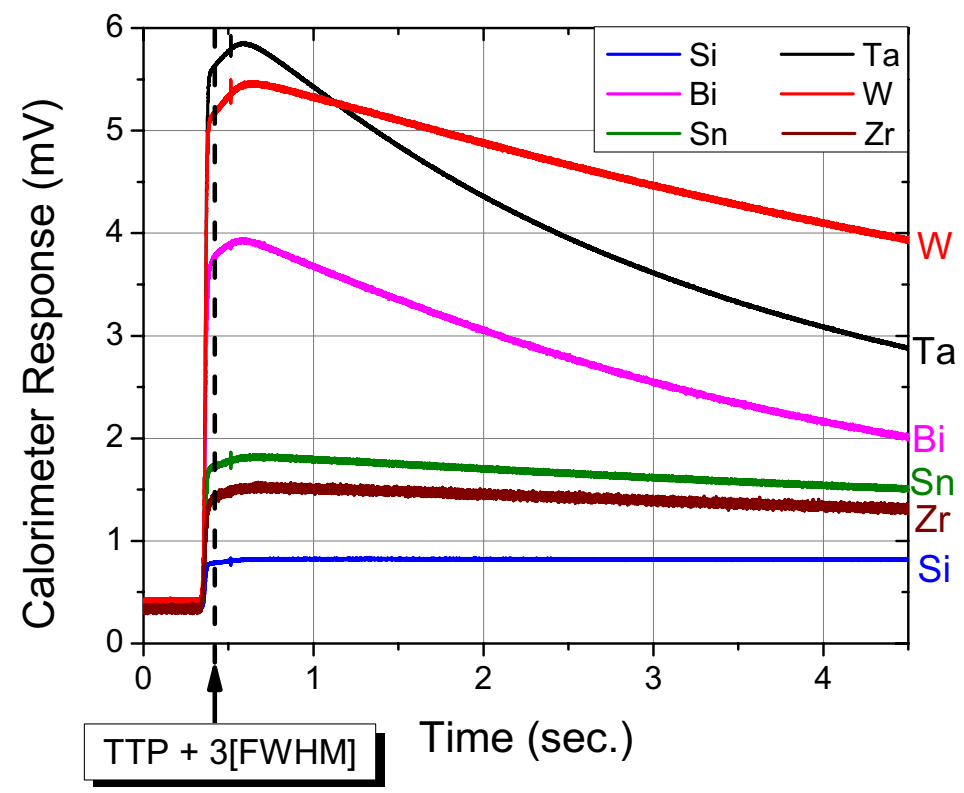

Figure 3. Time profile of bare calorimeter response following a $100 \mathrm{MJ}$ shot in the Lead/Poly bucket.

\section{Results and Discussion}

\subsection{Experiment}

The basic output from the calorimeters was the potential difference (in volts) as a function of time, sampled at a rate of $1 \times 10^{5}$ per second. Figure 3 illustrates a representative set of calorimeter outputs following a $100 \mathrm{MJ}$ ACRR pulse. For the purpose of evaluating the pulse-induced heating, the calorimeter response was assessed at a reference time of three full-width half-maxes (FWHMs) after the time to peak pulse (TTP), the shape and timing of which were acquired by means of a cadmium-based in-core self-powered neutron detector. This reference time was selected in order to minimize the impact of delayed gammas emitted from fission products accumulated during the pulse. The reference time does not, in general, coincide with the time of maximum calorimeter response.

As expected, the magnitude of the calorimeter response is correlated positively with the atomic number of the disc material, since photoelectric interactions constitute the primary means of energy deposition. Direct energy deposition from neutrons (i.e. the energy imparted to recoiling nuclei) can play a significant role at lower atomic masses but is negligible in comparison to the direct photon contribution at high atomic masses, mainly due to simple kinematic considerations. The fact that the responses in tantalum and tungsten exceed the bismuth response by a margin of $30-50 \%$, in spite of similarities in atomic number and heat capacity, warrants further elaboration, however.

To illuminate the source of the incongruity, the response of the bare calorimeter array is contrasted with the response of the cadmium-covered array in Fig. 4. The presence of the cadmium wrapper altered the radiation environment at the calorimeters in two ways:

1. By attenuating out impinging slow neutrons $(<0.5 \mathrm{eV})$ that would ordinarily instigate thermal, capture-type reactions within the calorimeters, and

2. By greatly enhancing the gamma fluence experienced by the calorimeters due to $(n, \gamma)$ reactions in Cd-113, without severely perturbing the impinging reactor $\gamma$ field. 


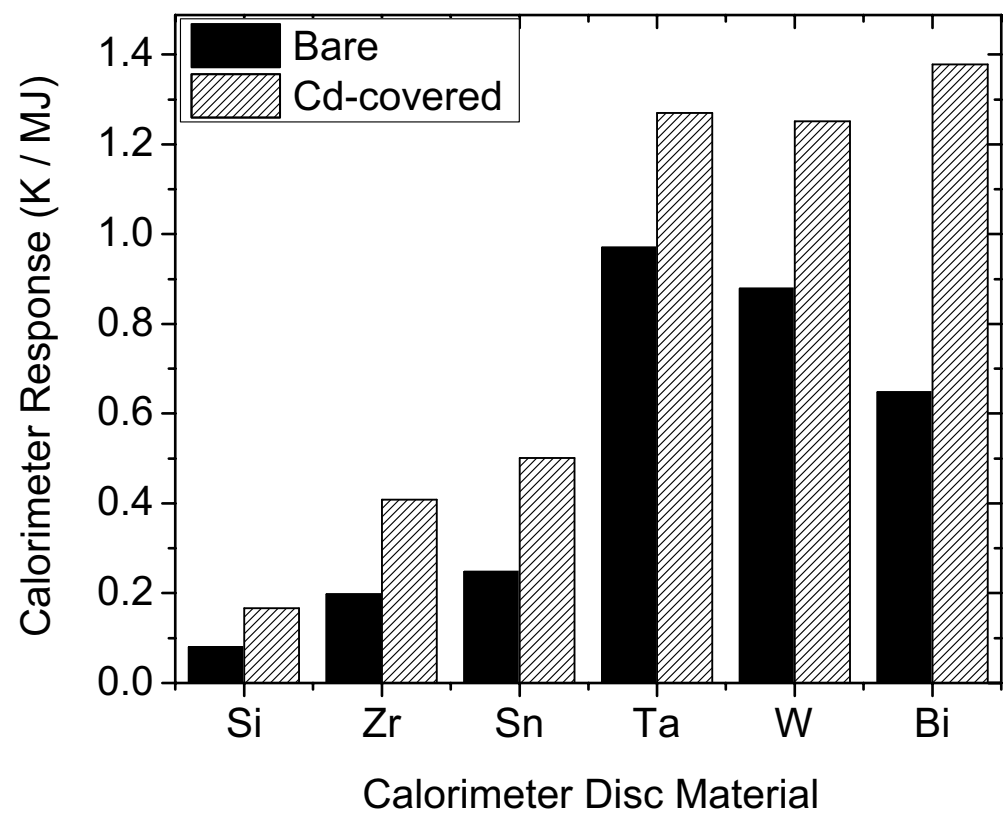

Figure 4. Mean heating of calorimeter discs per MJ as measured at TTP +3 (FWHM). The "Bare" and "Cd-covered" responses represent averages over 7 pulses and 3 pulses respectively in the range of 20-150 MJ.

From Fig. 4, it is apparent that the presence of the cadmium wrapper results in approximately a $2 \mathrm{X}$ augmentation of the calorimeter heating per $\mathrm{MJ}$ in $\mathrm{Si}, \mathrm{Zr}$, Sn, and $\mathrm{Bi}$. The degree of enhancement was considerably smaller in Ta and $\mathrm{W}$, which are distinguished from the other elements mainly by their large capture cross sections for low-energy neutrons. Because each calorimeter, by symmetry, experiences a nearly identical environment of impinging radiation, this implies that the difference in the enhancement factor lies in low-energy neutron interactions within the active disc, which is the only material component that varied among the calorimeters. Noting further that the direct energy deposition from low-energy neutron interactions is quite small (i.e. tens to hundreds of $\mathrm{eV}$ from capture-induced recoils, and essentially negligible from elastic scattering below the Cd cutoff) and also that the half-lives of relevant capture-generated activation products exceed the reference time of the calorimeter readings by several orders of magnitude, the difference in the enhancement factor is most plausibly explained by prompt capture gammas that are produced in the active discs and absorbed almost instantaneously within the same discs. To investigate this hypothesis further, the calorimeter response components were modeled in MCNP [3].

\subsection{Simulation}

Calculations of the neutron and gamma spectra at the location of the package were performed using an existing MCNP model of the ACRR [4]. In subsequent computations of the calorimeter response without the cadmium wrapper, the full reactor model was replaced with a surface source that bombarded the package with particles of the same spectral intensity as the actual reactor model. This simplification, which greatly reduced the computational burden of the response evaluations and allowed for efficient surveying of calorimeter internal $(n, \gamma)$ source contributions, relies upon two assumptions: (1) isotropy of impinging particle motion in the vicinity of the package, and (2) negligible perturbation of the ambient radiation field by the package. Since the aforementioned assumptions are invalid in the presence of the cadmium wrapper, all results pertinent to that setup were derived from the full reactor model. 

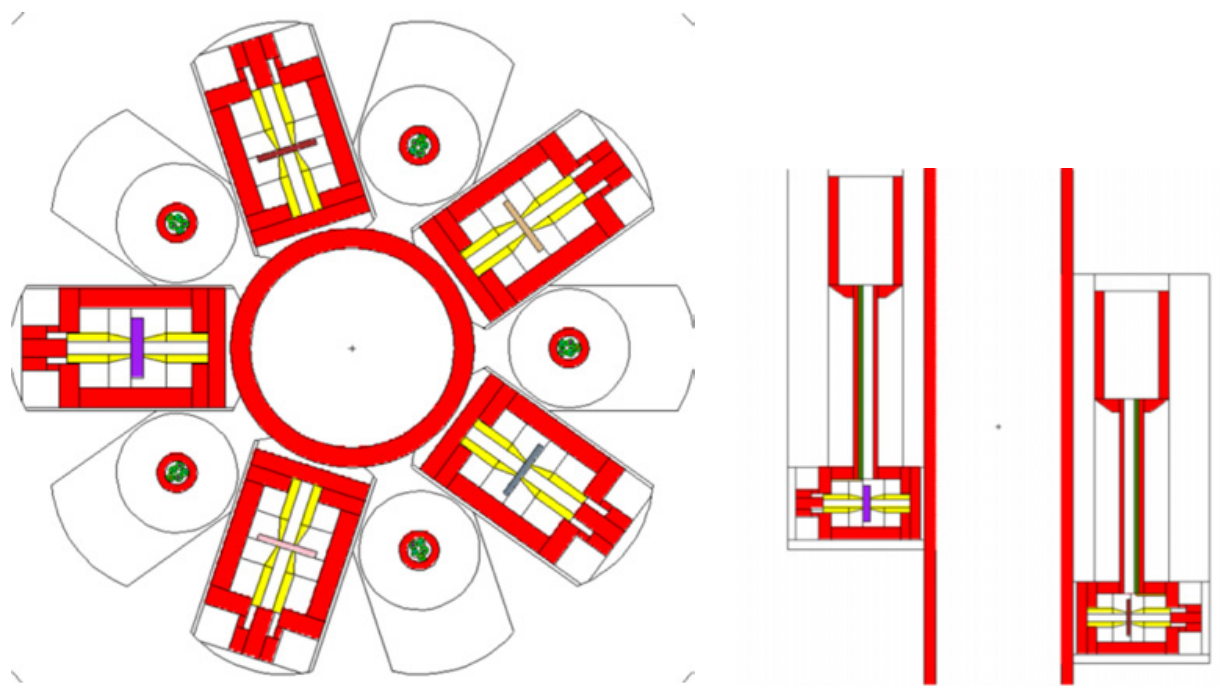

Figure 5. Axial (left panel) and lateral (right panel) cross sections of the modeled calorimeter assembly.

The model of the calorimeter assembly itself, shown in Fig. 5, included the active discs, alumina spacers, thermocouple wires and tubing, bolts and screws, and aluminum substructure of each calorimeter. ENDF/B-VI and B-VII cross sections were employed for all constituents of the calorimeter materials except tin and zinc, for which older ENDL libraries were utilized due to a lack of gamma production data in the more recent libraries.

The desired response metric is the total energy deposition in the active disc per megajoule of reactor pulse energy, which may be derived from the basic MCNP energy deposition tally using the following conversion:

$$
\frac{1 \mathrm{rad}}{\text { source }-\mathrm{n}} \cdot\left(\frac{2.4 \text { source }-\mathrm{n}}{\text { fission }}\right)\left(\frac{\text { fission }}{180 \mathrm{MeV}}\right)\left(\frac{1 \mathrm{MeV}}{1.6 \times 10^{-19} \mathrm{MJ}}\right)=8.33 \times 10^{16} \frac{\mathrm{rad}}{\mathrm{MJ}}
$$

where the first term in Eq. (1) represents the mean specific energy deposition in the active disc per fission-generated neutron, as sampled from the equilibrium (fundamental mode) spatial distribution of fissions in the reactor fuel. The adiabatic temperature rise of the disc is then ascertainable by dividing Eq. (1) by the specific heat capacity at constant pressure.

Calculated versus experimental values of the calorimeter heating per MJ in the lead / poly bucket are displayed in Table 1. Comparison reveals no systematic differences between the calculated and measured factors. Agreement is to within $10 \%$ in all materials, and, importantly, the impact of the cadmium wrapper - as evidenced by the Cd:bare response ratio in Table 1 - is reproduced very accurately in the MCNP model. Notably, the degree of suppression of the modeled Cd:base response ratio in high $(\mathrm{n}, \gamma)$ yield elements (Ta and $\mathrm{W}$ ) is nearly identical. This suggests that the model is correctly capturing the production, transport, and deposition of prompt $(\mathrm{n}, \gamma)$ radiation within the calorimeter array. In the process of performing the MCNP calculations, it was found that the *f8 (pulse height) tally produced more accurate heating factors than the $\mathrm{f} 4$ (track length estimator of flux) tally as folded with a kerma function. Specifically, heating factors generated with the $\mathrm{f} 4$ tally systematically exceed measured values by an average of $10 \%$, except in silicon. The difference in tally behavior is believed to be due to an absence of charged-particle equilibrium within the discs, which causes the kerma function to deviate from the true dose function. Under those conditions, the $* f 8$ tally in conjunction with electron transport provides a superior estimate of the dose. 
Table 1. Heating per MJ of pulse energy inside the lead/polyethylene bucket.

\begin{tabular}{|c|c|c|c|c|c|c|}
\hline \multirow{2}{*}{ Disc type } & \multicolumn{2}{|c|}{ EXPT. Heating (K / MJ) } & \multicolumn{3}{c|}{ CALC. Heating (K / MJ) } \\
\cline { 2 - 7 } & Bare & Cd-wrapped & $\begin{array}{c}\text { Ratio } \\
\text { (Cd:Bare) }\end{array}$ & Bare & Cd-wrapped & $\begin{array}{c}\text { Ratio } \\
\text { (Cd:Bare) }\end{array}$ \\
\hline $\mathbf{S i}$ & 0.081 & 0.167 & 2.1 & 0.075 & 0.158 & 2.1 \\
\hline $\mathbf{Z r}$ & 0.198 & 0.408 & 2.1 & 0.185 & 0.407 & 2.2 \\
\hline $\mathbf{S n}$ & 0.248 & 0.501 & 2.0 & 0.228 & 0.510 & 2.2 \\
\hline $\mathbf{T a}$ & 0.970 & 1.270 & 1.3 & 1.002 & 1.244 & 1.2 \\
\hline $\mathbf{W}$ & 0.878 & 1.252 & 1.4 & 0.806 & 1.222 & 1.5 \\
\hline $\mathbf{B i}$ & 0.648 & 1.378 & 2.1 & 0.654 & 1.402 & 2.1 \\
\hline
\end{tabular}
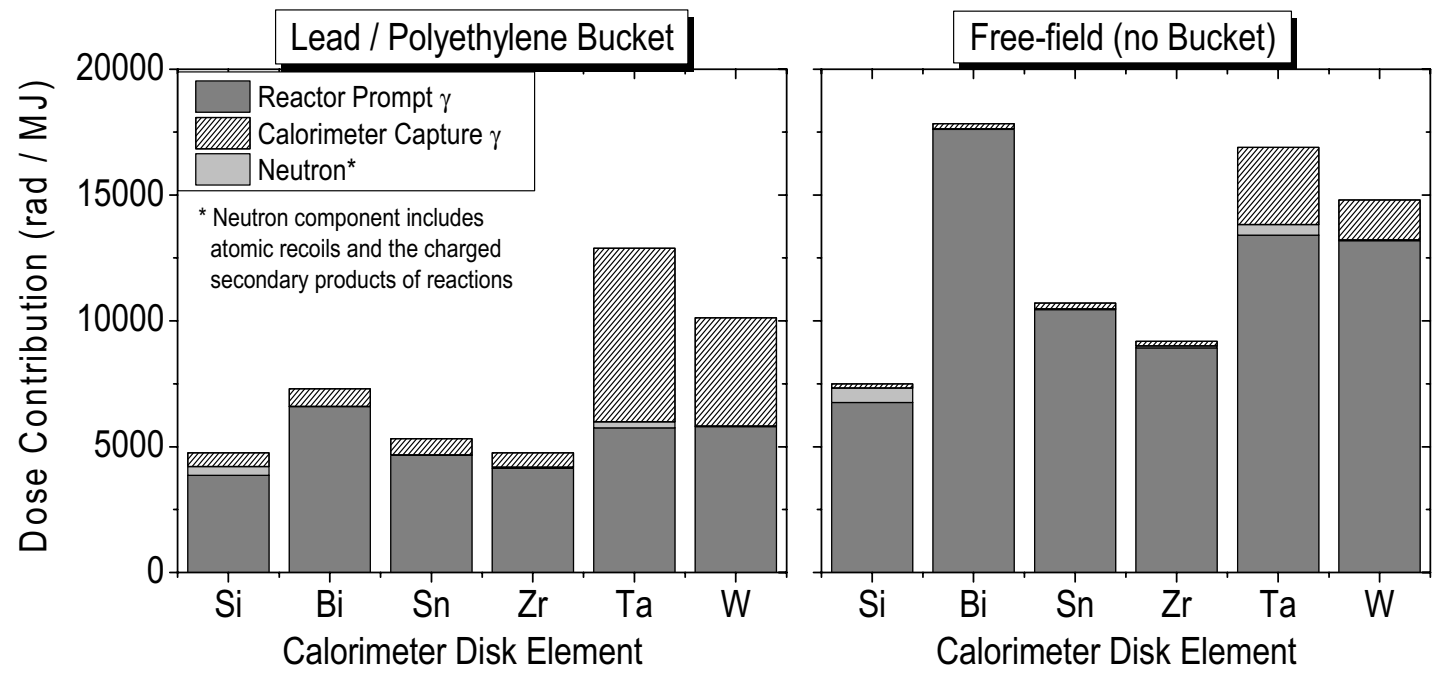

Figure 6. Breakdown of radiological heating contributions in the simulated, bare calorimeters.

\subsection{Analysis of Dose Origins}

With the basic modeling capability validated, the respective weights of the major radiative components of calorimeter heating were then investigated. This was accomplished by employing the surface source methodology discussed in Sect. 3.2 to model the incident neutron and gamma radiation separately, thereby allowing for a convenient accounting of the "direct" neutron and prompt $\gamma$ doses from the reactor as well as the $\gamma$ dose from prompt capture gammas generated within the calorimeter materials. A breakdown of the dose components is illustrated in Fig. 6.

Incident gammas from the reactor typically constitute the dominant contribution to calorimeter heating; however, the MCNP model predicts that neutron-induced capture reactions inside the calorimeter can account for over 50\% of the heating response in the well-thermalized environment of the lead-poly bucket and almost $20 \%$ of the response in free-field (with no bucket). The origins of the capture $\gamma$ response are further elucidated in Table 2, which shows the fractional contributions of the various calorimeter structural subcomponents to the total response in a bismuth disc. 
Table 2. Calorimeter subcomponent $(\mathrm{n}, \gamma)$ contributions to total heating response in bismuth disc.

\begin{tabular}{|c|c|c|}
\hline & $\begin{array}{c}\text { Lead / Poly } \\
\text { bucket }\end{array}$ & Free-field \\
\hline Aluminum body & $4.3 \%$ & $0.7 \%$ \\
\hline Steel screws & $0.3 \%$ & $<0.1 \%$ \\
\hline Thermocouple wires & $1.8 \%$ & $0.2 \%$ \\
\hline
\end{tabular}

Table 2 - generated via analysis of the response differentials caused by voiding out specific regions of the MCNP calorimeter model - reveals that the aluminum body and thermocouple wires are the most significant $(n, \gamma)$ contributors outside the disc, responsible for roughly $4 \%$ and $2 \%$ of the dose respectively within the lead/poly bucket. These non-disc contributions are effectively negligible in the nominal, free-field cavity.

\section{Conclusions}

A series of pulsed irradiations have been performed at ACRR for the purpose of characterizing the responses of elemental $\mathrm{Si}, \mathrm{Zr}, \mathrm{Sn}, \mathrm{Ta}, \mathrm{W}$, and $\mathrm{Bi}$ calorimeters in the nominal test cavity as well as in the highly thermalized environment generated by insertion of a lead/polyethylene bucket into the cavity. By comparing the measured heating responses at a reference time of three FWHM after the pulse peak - with and without a 30 mil cadmium wrapper around the test assembly - it was argued that prompt secondary gammas produced inside the active calorimeter discs and absorbed within the same discs were responsible for a considerable portion of the response in a well-thermalized neutron environment. The measured heating factors were corroborated through MCNP analysis of the experimental setup, which confirmed major trends in the data and successfully reproduced heating factors to within $10 \%$.

Subsequent computational analysis of the apportionment of radiative contributions to the heating response indicated that $(\mathrm{n}, \gamma)$ capture reactions internal to the calorimeter were responsible for $40 \%$ of the heating in tungsten, $50 \%$ of the heating in tantalum, and under $10 \%$ in all other elements when irradiated in the lead/poly bucket. Even in the nominal ("free-field") cavity environment, which is more epi-thermal, calorimeter-internal neutron capture reactions were found to contribute as much as $20 \%$ to the total response. These results suggest that care must be taken to account for the prompt $(\mathrm{n}, \gamma)$ dose when utilizing certain calorimeter types for auxiliary dosimetry purposes, as such reactions can cause a substantial local incongruence in the radiation environment relative to nearby test articles.

\section{References}

[1] S. M. Luker, P. J. Griffin, K. R. DePriest, D. B. King, G. E. Naranjo, J. S. Suo-Anttila and N. Keltner, J. ASTM Int., 3, 100380 (2006)

[2] S. M. Luker, P. J. Griffin, K. R. DePriest, D. B. King, G. E. Naranjo and J. S. Suo-Anttila, J. ASTM Int., 4, 100376 (2007)

[3] X-5 Monte Carlo Team, Technical Report LA-UR-03-1987, Los Alamos National Laboratory, Los Alamos, NM (2003, revised 2008)

[4] K. R. DePriest, P. J. Cooper and E. J. Parma, Technical Report SAND2006-3067, Sandia National Laboratories, Albuquerque, NM (2006) 